\title{
Improved cortical activity and reduced gait asymmetry during poststroke self-paced walking rehabilitation
}

\author{
Keonyoung Oh ${ }^{1,2}$, Jihong Park ${ }^{3}$, Seong Hyeon Jo², Seong-Jin Hong ${ }^{2}$, Won-Seok Kim³ ${ }^{3}$ Nam-Jong Paik ${ }^{3 *}$ and \\ Hyung-Soon Park $^{2^{*}}$ (D)
}

\begin{abstract}
Background: For patients with gait impairment due to neurological disorders, body weight-supported treadmill training (BWSTT) has been widely used for gait rehabilitation. On a conventional (passive) treadmill that runs at a constant speed, however, the level of patient engagement and cortical activity decreased compared with gait training on the ground. To increase the level of cognitive engagement and brain activity during gait rehabilitation, a self-paced (active) treadmill is introduced to allow patients to actively control walking speed, as with overground walking.
\end{abstract}

Methods: To validate the effects of self-paced treadmill walking on cortical activities, this paper presents a clinical test with stroke survivors. We hypothesized that cortical activities on the affected side of the brain would also increase during active walking because patients have to match the target walking speed with the affected lower limbs. Thus, asymmetric gait patterns such as limping or hobbling might also decrease during active walking.

Results: Although the clinical test was conducted in a short period, the patients showed higher cognitive engagement, improved brain activities assessed by electroencephalography (EEG), and decreased gait asymmetry with the self-paced treadmill. As expected, increases in the spectral power of the low $\gamma$ and $\beta$ bands in the prefrontal cortex (PFC), premotor cortex (PMC), and supramarginal gyrus (SG) were found, which are possibly related to processing sensory data and planning voluntary movements. In addition, these changes in cortical activities were also found with the affected lower limbs during the swing phase. Since our treadmill controller tracked the swing speed of the leg to control walking speed, such results imply that subjects made substantial effort to control their affected legs in the swing phase to match the target walking speed.

Conclusions: The patients also showed reduced gait asymmetry patterns. Based on the results, the self-paced gait training system has the potential to train the symmetric gait and to promote the related cortical activities after stroke. Trial registration Not applicable

Keywords: Gait training, Self-paced treadmill, Stroke rehabilitation, Electroencephalography, Gait asymmetry, Cortical activation

\footnotetext{
*Correspondence: njpaik@snu.ac.kr; hyungspark@kaist.ac.kr

2 Department of Mechanical Engineering, Korea Advanced Institute of Science and Technology (KAIST), 291 Daehak-ro, Yuseong-gu,

Daejeon 34141, Republic of Korea

${ }^{3}$ Department of Rehabilitation, Seoul National University Bundang

Hospital, 82, Gumi-ro 173 Beon-gil, Bundang-gu, Seongnam-si, Gyeonggi-do 13620, Republic of Korea

Full list of author information is available at the end of the article
}

\section{Background}

Stroke is a prevalent disease caused by hemorrhagic or ischemic injury in the brain and accompanied by motor disability. Impaired motor function can be recovered to some extent by cortical reorganization [1]. Rehabilitation within the first 3 months after stroke is essential to original author(s) and the source, provide a link to the Creative Commons licence, and indicate if changes were made. The images or other third party material in this article are included in the article's Creative Commons licence, unless indicated otherwise in a credit line to the material. If material is not included in the article's Creative Commons licence and your intended use is not permitted by statutory regulation or exceeds the permitted use, you will need to obtain permission directly from the copyright holder. To view a copy of this licence, visit http://creativecommons.org/licenses/by/4.0/. The Creative Commons Public Domain Dedication waiver (http://creativeco mmons.org/publicdomain/zero/1.0/) applies to the data made available in this article, unless otherwise stated in a credit line to the data. 
promote recovery by neural plasticity [2]. The restoration of locomotor function is a major issue in rehabilitation for many patients affected by stroke since gait disorders limit performing social and daily living activities [3]. In addition, the risk of falls that could cause traumatic injury increases as gait performance decreases [4]. Thus, in the hospital and afterwards, gait rehabilitation is provided for stroke survivors to improve their walking performance $[5,6]$.

Body weight-supported treadmill training (BWSTT) is widely performed for gait rehabilitation since it provides safe and repeatable training in small spaces with less burden on the therapist compared with overground training. However, there is a study showing that the effect of training on a treadmill was not superior to that of overground training with a therapist [7]. On the conventional treadmill, patients have difficulties voluntarily participating and engaging in training because they adapt to the unchanging speed. To overcome this limitation of the traditional treadmill, a self-paced treadmill that simulates overground walking was developed [8-11], and sometimes with a virtual reality system $[12,13]$ for gait rehabilitation after neurological disorders [10, 12, 13]. The speed of the treadmill belt is controlled by preferences of a user by measuring the position of the user's body segments [11, 14] using a commercial depth sensor [15] or using a motion capture system [16]. In addition, unexpected inertial force by sudden acceleration and deceleration was modified by the velocity of the swing foot [9].

Several studies reported differences in a variability of spatiotemporal gait parameters between the self-paced treadmill walking and the conventional treadmill walking with a fixed speed. A larger variability of stride length and stride time was found with the self-paced treadmill than the conventional treadmill in neurologically intact human subjects $[17,18]$. When a control algorithm of the self-paced treadmill is adjusted to be more sensitive to a user's movement, larger variabilities of these gait parameters are found [19]. In addition, such spatiotemporal gait parameters on self-paced treadmill can be effectively used for Multiple Sclerosis prediction [20]. As far as the authors know, however, it remains unclear how the self-paced treadmill walking affects cortical activities in patients after neurological disorders.

Monitoring the brain activity of the patient is helpful in identifying the effect of training and suggests an effective gait rehabilitation approach since impaired function could be recovered by cortical reorganization [21]. Several studies have shown that the function and structure in the regions adjacent to the impaired area after stroke, as well as regions remote from the cortical lesion, were changed during the recovery process by brain plasticity [22]. In addition to the voluntary recovery of lost function during the initial several months after stroke, therapy-induced neural reorganization could promote recovery $[23,24]$.

There are several noninvasive neuroimaging techniques to monitor cortical activity, such as fMRI, fNIRS, and EEG. Functional magnetic resonance imaging (fMRI) is widely used to elucidate brain activation due to its high spatial resolution. Although the activity in the brain, including deep brain tissue and the cerebral cortex, can be monitored by fMRI, this neuroimaging technology is not suitable to study cortical activity during dynamic walking since the subject has to remain in the lying position in a static state before or after the intervention [25]. To monitor cortical activity during dynamic activities, functional near-infrared spectroscopy (fNIRS) and electroencephalography (EEG) are suitable to examine cortical changes during walking. Increased oxygenated hemoglobin (oxyHb) in sensorimotor cortices and supplementary cortex was monitored using fNIRS during walking $[26,27]$. In another study, activation in the prefrontal, premotor and sensorimotor cortex was reported to increase while walking [28]. During precise stepping, increased oxyHb in the prefrontal and supplementary motor cortex was observed in another study [29]. In research with participants performing a dual task during walking, a change in activity in the prefrontal cortex related to cognitive performance was observed [30].

Although EEG recording has a low spatial resolution, intra-stride characteristics could be identified due to its high temporal resolution. Previous studies have reported that spectral power decreased in the $\mu$ and $\beta$ bands in the sensorimotor cortex are observed during movement $[31,32]$. These characteristics are referred to as eventrelated desynchronization (ERD). One study elucidated that the intra-stride patterns of cortical activity in the anterior cingulate, posterior parietal, and sensorimotor cortex were coupled to the gait phase during steady walking on a treadmill [28]. In another study, significant ERD in the $\beta$ band and low $\gamma$ band was demonstrated during active walking [33]. EEG can also be used to demonstrate effective connectivity by calculating Granger causality $[34,35]$. In one study, connectivity between motor areas and others decreased, but connectivity between nonmotor areas increased during steady walking compared to standing [36].

In this study, we used a similar experimental protocol as that used with healthy subjects in the previous study to extend the analysis to stroke survivors [33]. Brain activity levels were monitored and compared between the selfpaced treadmill and conventional treadmill with the fixed walking speed using EEG recording. We hypothesized that active walking on the self-paced treadmill allows patients to engage more in the training and to increase 
cortical activity than walking at a fixed speed. In addition, gait asymmetry patterns might decrease during active walking, as target walking speed is actively maintained, compared to passive walking conditions.

\section{Methods}

Participants with postischemic stroke hemiplegia who could provide consent and adhere to the study protocol without any apparent cognitive impairment were prospectively screened for the ability to walk without manual assistance and recruited from the Seoul National University Bundang Hospital (SNUBH), a tertiary teaching hospital located in Seongnam, Korea, from December 2016 to September 2017. They were excluded if they scored below three on the Functional Ambulation Categories (FAC) $[37,38]$. The institutional review board of SNUBH approved the study protocol (IRB no. B-1608/358-004), and written informed consent was obtained from all the participants.

The self-paced treadmill was controlled by recognizing a user's intention through acceleration or deceleration (Fig. 1) $[9,15]$. The positions and velocities of the user's pelvis and foot were measured using KINECT (Microsoft, Redmond, WA, US), and the data were processed by MFC (Microsoft, Redmond, WA, US). If a user wanted to walk faster and moved forward with a faster swing leg, the controller recognized this intention and accelerated the belt. For active walking, both the target speed and the current speed controlled by the user's intention of speed were displayed on the monitor, so the user could change their speed to adjust to the target speed.

All participants were evaluated with the FAC first, followed by the Berg balance scale (BBS) [39],
Falls Efficacy Scale (FES) [40] and 10-m walking test $(10 \mathrm{mWT})$, and relevant demographic data, such as age and medical history, were obtained through an interview conducted at the beginning of the session.

The participants performed two active and two passive walking sessions in random order. Each walking session consisted of a set of slow walking and fast walking trials, which were repeated nine times. Between each session, a minimum of a 5-min break was provided to avoid fatigue effects. EEG data were collected while participants performed the walking trials. For the safety of the subjects, the fast and slow speeds in the walking sessions were determined not to exceed the maximum walking speed measured from the $10-\mathrm{m}$ walking test and were set to $70 \%$ and $40 \%$ of the maximum walking speed from the 10 -m walking test.

To identify walking phases, four one-dimensional load cells (DSCK-500, Bongshin, Osan, South Korea) were implemented in each corner of the treadmill. From measured vertical ground reaction forces (GRF), instants of heel-strikes (HS) and toe-off (TO) were calculated when the GRF exceeded and dropped below a threshold of $20 \mathrm{~N}$. To investigate the gait asymmetry patterns, gait phase durations were also calculated from such gait indices. The GRF data were collected at $1080 \mathrm{~Hz}$ and then filtered with a low-pass filter with a cutoff frequency of $20 \mathrm{~Hz}$. Gait asymmetry was estimated by calculating the difference in stance duration between the two lower limb sides and then compared between the active and passive walking using the paired $\mathrm{t}$-test. Since the walking speed was fixed for the passive walking session with zero variance, the walking speeds between the active and passive walking conditions were compared using the Wilcoxon Rank Sum test.

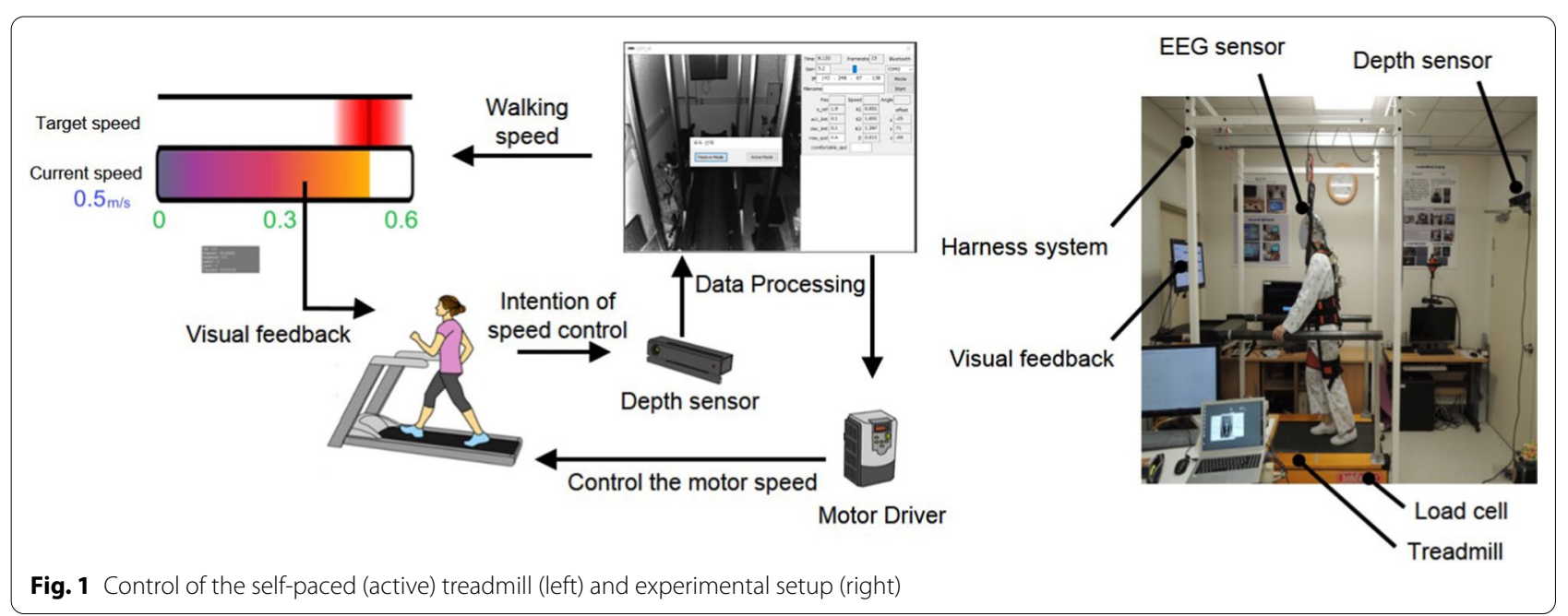




\section{Collecting EEG data and signal processing}

To assess the level of activity in each cortical region, EEG data were collected using actiCHamp (Brain Vision, Morrisville, NC, United States). A total of 64 channels were located on a subject's scalp, and the data were collected at $1080 \mathrm{~Hz}$. Postprocessing of EEG data using EEGlab (Swartz Center for Computational Neuroscience, Lo Jolla, CA, United States) based on MATLAB (Mathworks, Natick, MA, United States) toolbox was conducted. Details on the postprocessing procedures were adapted from and can be found in the previous research [33].

First, all the signals from the walking trials and the rest periods were combined in a single data set. A Butterworth 5th order high-pass filter was then applied with a cutoff frequency of $1 \mathrm{~Hz}$. To eliminate noisy data, signals containing a standard deviation (SD) larger than $1000 \mu \mathrm{V}$ or kurtosis larger than five SD were removed [28]. After removing noisy data, the EEG signals were rereferenced to have a common average value. To remove a signal contaminated with artifacts, an artifact subspace reconstruction (ASR) function [41] from the EEGlab was conducted. Compared to the relatively clean signals during the $2 \mathrm{~min}$ of rest without any kinematic change, a statistical test was conducted with a moving window $(500 \mathrm{~ms})$ of a single channel to find signals that have variance larger than a threshold of three SD, and such signals were identified as corrupted data.

Since any single channel could be affected by neighboring channels and nonbrain signals, an extended independent component analysis (EICA) [42-44] was conducted to determine the independent components (IC) (or signals) from only brain sources. After the Adaptive mixture independent component analysis (AMICA), equivalent current dipole sources were found using the DIPFIT function in EEGlab [45]. Dipoles accounting for less than $20 \%$ of the variance of the calculated IC projection on the scalp were neglected, and other dipoles were separated into nonbrain and brain sources according to their locations and power spectra. The clustering of ICs was then conducted for all the subjects using the power spectral density and the location of the dipole. A k-means clustering method was used to make the clusters, and ICs having variance larger than three SDs were identified as outliers and neglected for the analysis. The Brodmann areas of the estimated clusters were identified using the Talairach atlas [46].

To perform a temporal analysis of the EEG data, the EEG data were synchronized from the calculated gait phase indices. From all the points that were in the 0.1-s period before the right heel strike (RHS), epochs were obtained to have a minimum of one gait stride from the RHS to the following RHS. Such epochs were then categorized into four conditions: slow, fast, accelerated, and decelerated walking for both active and passive walking tasks. Within each epoch, a time-warping function was performed for all indices (RHS, LTO, LHS, RTO, and RHS) that have the same latencies from the start of the stride.

To investigate intra-stride cortical activity, the power spectral density with respect to each gait index, called event-related spectral perturbations (ERSP), was calculated. All the ERSPs from the ICs in a cluster were calculated first and then averaged to obtain the grand mean ERSP in a cluster. Each frequency component of such time-frequency decomposition was then normalized to an average value of the frequency component itself. A significance test and masking in the ERSP were conducted using a bootstrap function in the EEGlab.

To compare the cortical activities between active and passive walking, event-related desynchronization (ERD) and synchronization (ERS) were computed. Before comparing ERD/ERS in each cluster from the two conditions, the result from the time-frequency decomposition with the rest period dataset in the same cluster was subtracted to act as a baseline. A direct comparison of the level of activation was then conducted by calculating a difference in the ERD/ERS from two conditions. Statistical differences in ERSPs between the active and passive walking sessions were determined using the non-parametric bootstrapping method implemented in the EEGlab [43], with a significance level of 0.05 .

\section{Results}

Seven (four males and three females; $67.85 \pm 9.40$ years old) stroke survivors participated this study and were enlisted for the analysis (Table 2). The average slow and fast walking speeds during the active walking sessions were $0.28 \pm 0.14 \mathrm{~m} / \mathrm{s}$ and $0.42 \pm 0.15 \mathrm{~m} / \mathrm{s}$, respectively, and $0.25 \pm 0.00 \mathrm{~m} / \mathrm{s}$ and $0.42 \pm 0.00 \mathrm{~m} / \mathrm{s}$ during the passive walking session (Fig. 2). There was no significant difference in the walking speeds between the passive and active walking sessions.

The k-means clustering resulted in seven clusters (Fig. 3a and Table 1). The Brodmann area, numbers of ICs, and subjects in each cluster are represented in Table 1. ERSPs from all the clusters computed showed periodic fluctuations as the gait cycle passed (Fig. 3). The spectral power of the $\beta$ and $\gamma$ frequency bands from the PMC, PFC, and SG increased in the double support and early swing phase (Fig. 3).

Compared to the passive walking, the active walking aroused enhanced synchronization and desynchronization in some cortices. The lower $\gamma(30-50 \mathrm{~Hz})$ band desynchronizations in the PMC were significantly enhanced in the active walking trials (Figs. 4 and 5). 

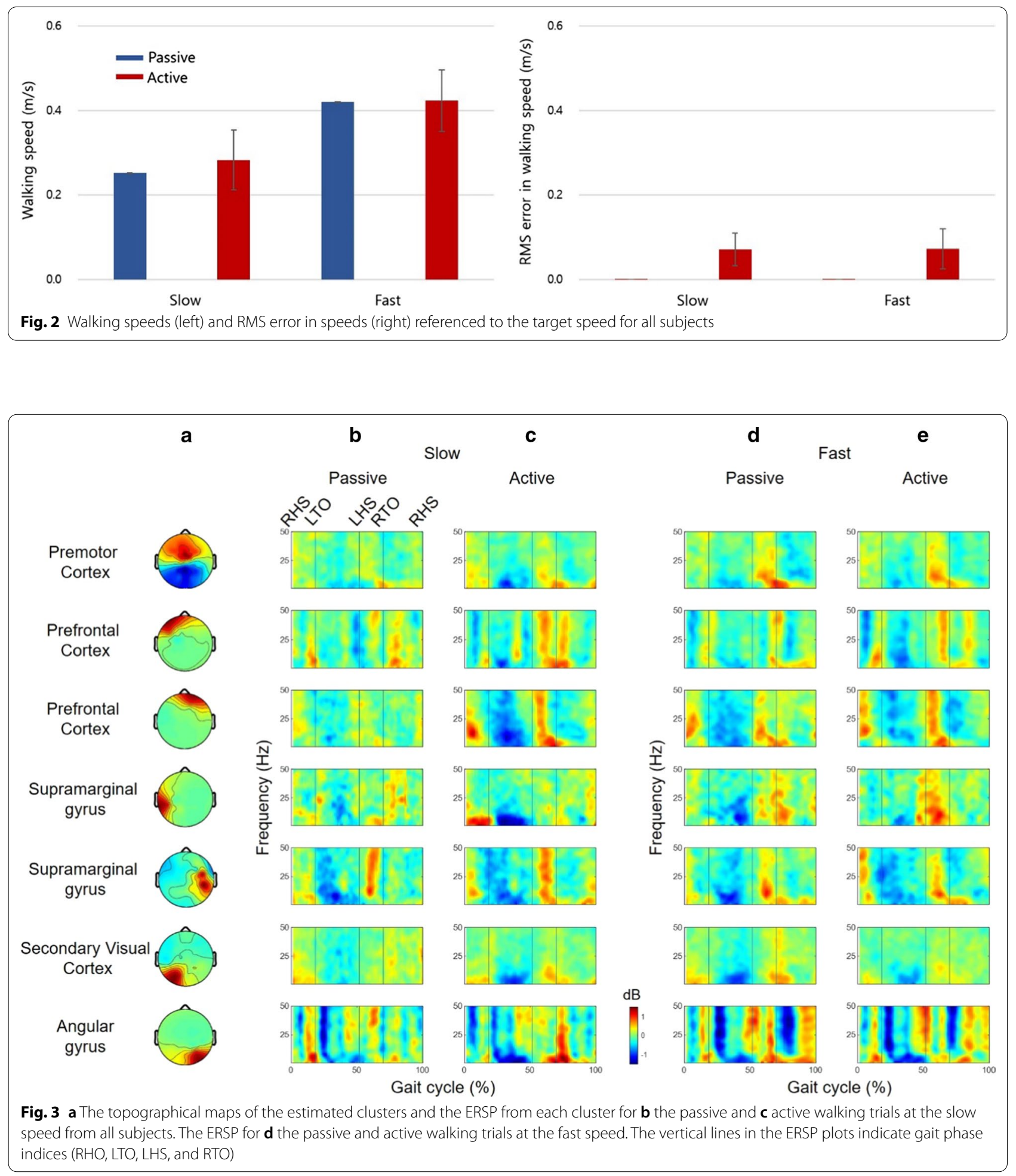

The upper $\beta(14-30 \mathrm{~Hz})$ band synchronizations were enhanced in the PFC and SG (Figs. 4 and 5). The $\mu$ band desynchronizations were enhanced in the SG and the visual cortex (Figs. 4 and 5).
Such changes in cortical activities were significant, even in the affected side of brain. The subjects with the lesion on the left side of their brain showed enhanced upper $\beta$ band synchronizations in the PFC and SG at 
Table 1 Calculated dipoles (electrical sources) and clusters

\begin{tabular}{|c|c|c|c|c|c|c|c|}
\hline $\begin{array}{l}\text { Name of } \\
\text { cluster }\end{array}$ & $\begin{array}{c}\text { Premotor } \\
\text { cortex }\end{array}$ & $\begin{array}{c}\text { Prefrontal } \\
\text { cortex }\end{array}$ & $\begin{array}{c}\text { Prefrontal } \\
\text { cortex }\end{array}$ & $\begin{array}{c}\text { Supramarginal } \\
\text { gyrus }\end{array}$ & $\begin{array}{c}\text { Supramarginal } \\
\text { gyrus }\end{array}$ & Visual cortex & Angular gyrus \\
\hline \multicolumn{8}{|l|}{ Dipole map } \\
\hline Number of ICs & 18 & 10 & 9 & 14 & 12 & 6 & 16 \\
\hline Subject \# & $\begin{array}{c}1,2,3,4,5,6 \\
7\end{array}$ & $3,4,5,6,7$ & $3,4,5,6,7$ & $1,3,4,5,6,7$ & $3,4,5,7$ & $3,5,7$ & $\begin{array}{c}1,2,3,4,5,6 \\
7\end{array}$ \\
\hline $\begin{array}{c}\text { Brodmann } \\
\text { Area \# }\end{array}$ & $\begin{array}{c}6 \text { (Medial } \\
\text { Frontal Gyrus) }\end{array}$ & $\begin{array}{c}8 \text { (Superior } \\
\text { Frontal Gyrus) }\end{array}$ & $\begin{array}{l}10 \text { (Superior } \\
\text { Frontal Gyrus) }\end{array}$ & $\begin{array}{c}40 \text { (Inferior } \\
\text { Parietal } \\
\text { Lobule) }\end{array}$ & $\begin{array}{c}40 \text { (Inferior } \\
\text { Parietal } \\
\text { Lobule) }\end{array}$ & 19 (Cuneus) & $\begin{array}{c}39 \text { (Inferior } \\
\text { Parietal } \\
\text { Lobule) }\end{array}$ \\
\hline
\end{tabular}

both walking speeds (Fig. 5c, d and Table 2). The subjects with the lesion on the right side of their brain also showed significantly enhanced $\beta$ band synchronizations in the PFC (Fig. 5e, $\mathrm{f}$ and Table 2).

Gait asymmetries due to hemiparesis also decreased during the active treadmill trials. The participants showed decreased asymmetry by an average of $32.0 \%$ and $25.7 \%$ during slow $(\mathrm{p}=0.032)$ and fast $(\mathrm{p}=0.057)$ walking speeds, respectively (Fig. 6).

\section{Discussion}

The stroke survivors on the self-paced treadmill described above showed increased cortical activities in their brains compared to the conventional passive treadmill. Such cortical changes were significant in the visual cortex, premotor cortex, supplementary motor cortex, and somatosensory cortex, especially when the affected lower limb was in the late-swing phase and immediately before its heel strike. Since the controller implemented in our active treadmill system tracked the speed of the swing leg to estimate the user's gait speed and to set the target speed, the increases in cortical activation level implied that subjects paid substantial attention to the target speed and tried to adjust the speed of their affected legs in the swing phase to match the target walking speed given by the self-paced treadmill system. Possible explanations of the increased levels of cortical activation in each region of cortex is further discussed below.

\section{Activation in the prefrontal cortex}

The prefrontal cortex showed a higher activation level during active walking, and it could be presumed that subjects paid more attention to processing visual feedback on the velocity error and trying to maintain the given target speed as instructed. The processing of visual feedback and the corresponding greater control of their body joints might have imposed an additional task for the participants. Based on previous studies that reported that the prefrontal cortex might be relevant to gaze orientation, error detection, sensory reweighting and feedback, and executive functions, the subjects seemed to try to pay attention to performing such tasks. During human balance control with external perturbation, greater activation in the PFC was found and interpreted as an effort to assess the error through visuospatial attention [47]. To detect error between the target and the subject's speed, similar cortical involvement processing visuospatial information might be engaged. All the subjects who participated in this experiment understood the task and performed well, so it is possible to speculate that the subjects did not have severe injury in the PFC. Because the PFC may also contribute to orchestrating internal goals and means [48], if the PFC is impaired after stroke, the subjects might find it difficult to understand and repeatedly perform the two different tasks. Moreover, the PFC is also known to carry out cross-modal sensory processing, and such involvement could be seen if we look at the EEG signal within the gamma band. During active walking, such a gamma band showed a higher activation level, so it may be relevant to combining visual, spatial, and possibly other sensory information. 


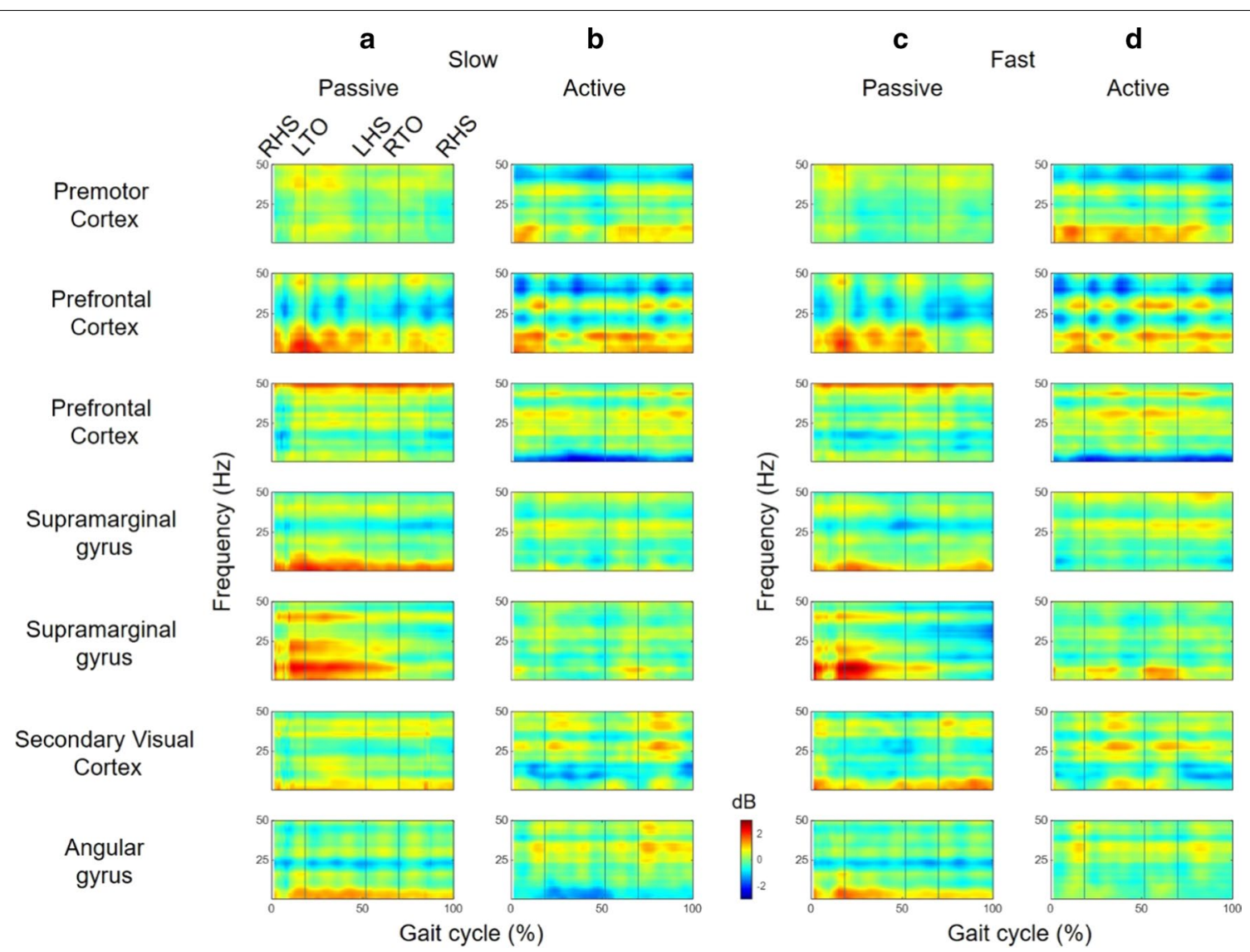

Fig. 4 The ERD/ERS (active-passive) from the estimated clusters during a stride from all subjects for $\mathbf{a}$ the passive and $\mathbf{b}$ active walking trials at the slow walking speed and $\mathbf{c}$ passive and $\mathbf{d}$ active walking trials at the fast walking speed. The vertical lines in the ERSP plots indicate gait phase indices (RHO, LTO, LHS, and RTO)

\section{Activation in the supplementary motor area}

Following cortical involvement in the PFC, which assesses visuospatial information, the involvement and higher activation levels in the supplementary motor cortex might be relevant to conducting tasks by programming sequential and complex locomotor mechanisms. Recently, a number of studies have been conducted to reveal the role of the SMC, and these studies have suggested that the SMC could provide a bridge between the intention in mind and voluntary external movement [49] and could organize motor behavior [50]. Significant activation in the SMC was found before movement in the fingers of human subjects, and the activation preceded cortical involvement in the PMA, which is known to control actual movements [51]. Rather than the posterior part of the SMC, it was the anterior part that was relevant to preparing for the motion. Once the subject was asked to follow the target speed, they had to assess the error between their actual speed and the target speed and decide whether they needed to accelerate or decelerate. Differing from steady-state (or fixed-speed) walking, the subjects might be asked to make decisions regarding such transient gait speed and prepare subsequent movement, including faster/slower swing of the leg or larger/smaller magnitude of propulsion forces. Such preparation for sequential movements may be related to greater activation in the SMC. Some researchers have suggested that the SMC also played a role in preparing and performing complex movements, such as gait initiation [52], which could be supported by our results that the SMC was highly activated when the subjects were asked to prepare and perform a relatively complex transient gait rather than steady walking.

\section{Cortical involvement of the somatosensory cortex}

The patients engaged greater cortical effort to process sensory information from their limbs during active walking than during passive walking. Although both speeds were not significantly different during passive and active walking tasks (Fig. 2), the belt speed was controlled by tracking the pelvis position and the speed of the swing leg, so the subjects needed to control their lower limbs 


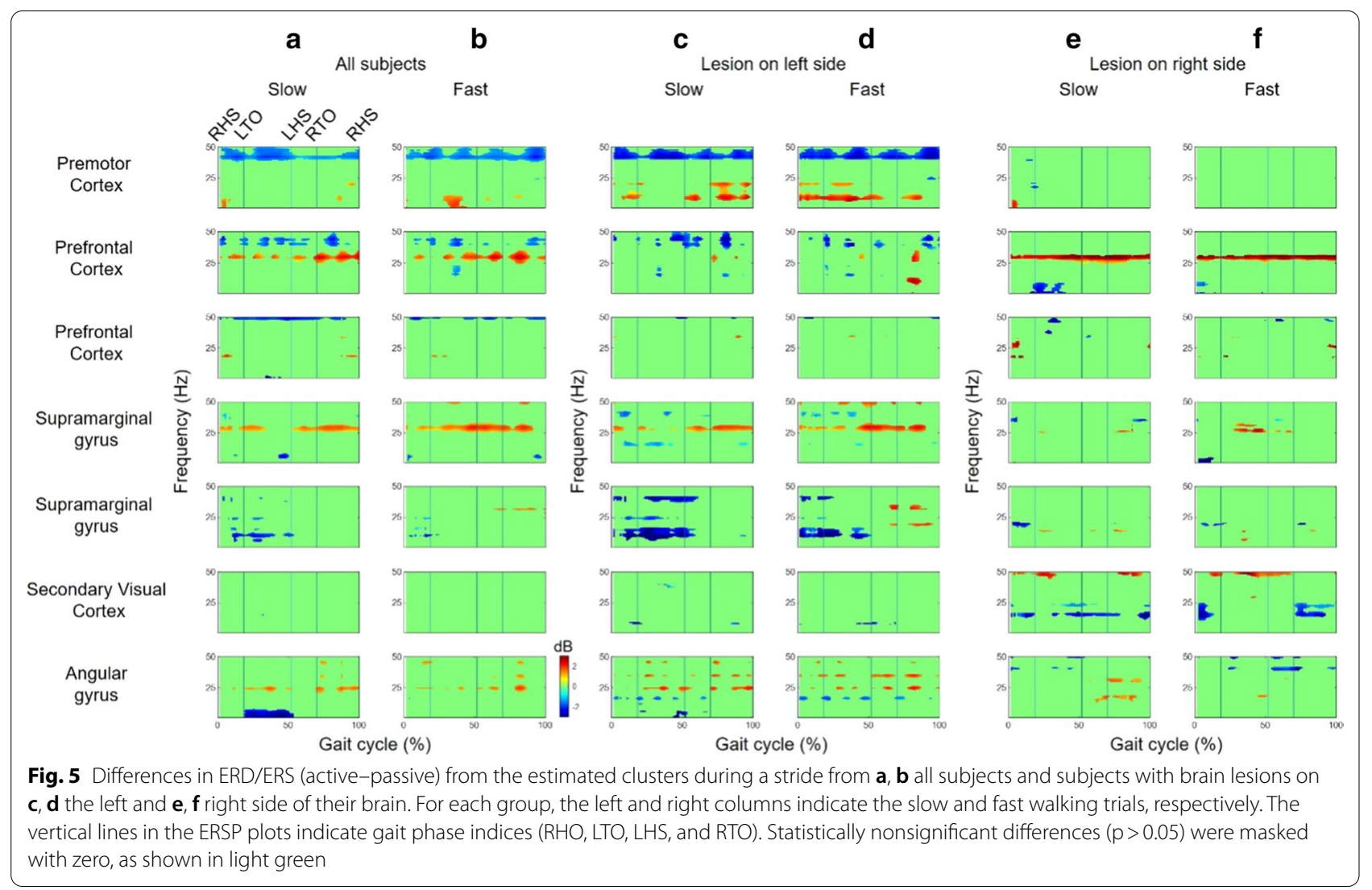

Table 2 Demographics and clinical scores for the poststroke hemiplegic patients (ACA anterior cerebral artery, MCA middle cerebral artery, PCA posterior cerebral artery)

\begin{tabular}{|c|c|c|c|c|c|c|c|c|}
\hline $\begin{array}{l}\text { Subject } \\
\text { number }\end{array}$ & Sex & Age & Location of infarcts & $\begin{array}{l}\text { Time since stroke } \\
\text { onset }\end{array}$ & FAC & BBS & FES & $10 \mathrm{mWT}$ \\
\hline 1 & Female & 60 & Left MCA territory & 13 days & 5 & 56 & 65 & 11.7 \\
\hline 2 & Male & 66 & Multiple lacunes & 2.5 years & 4 & 52 & 89 & 24.0 \\
\hline 3 & Male & 84 & Left MCA and ACA territory & 21 days & 4 & 46 & 106 & 11.87 \\
\hline 4 & Female & 75 & Right MCA territory & 26 years & 5 & 56 & 82 & 15.67 \\
\hline 5 & Male & 73 & Right MCA territory & 13 days & 3 & 49 & 60 & 11.87 \\
\hline 6 & Female & 54 & Left PCA territory & 11 days & 5 & 55 & 4 & 8.08 \\
\hline 7 & Male & 63 & Left MCA territory & 9 days & 3 & 55 & 13 & 6.74 \\
\hline
\end{tabular}

precisely to match the given target speed. Oscillatory power in the beta band was also increased in the somatosensory cortex in the supramarginal gyrus, which implied that there was an increase in neural populations recruited during active walking. To date, several studies have reported that the somatosensory cortex in the supramarginal gyrus contributes to body positioning, especially upright positioning [53], finger positioning [54], and body displacement perceptions [55]. In addition, the $\beta$ band $(13-30 \mathrm{~Hz})$ in the sensorimotor cortex has been shown to become activated when movements occur [31, 56, 57]. Moreover, such $\beta$ band power and connectivity between the sensorimotor cortex and the parietal cortex were shown to have larger sensory feedback gain, as it was very important to perform accurate movement [58]. As movement selection demands vary, the cortex may distribute the activity of the neural population $[59,60]$ in alpha and beta bands. To distinguish the role of alpha and beta band activities in the sensorimotor cortex from each other, a virtual task that involved imagining grasping a bar was conducted, and a larger population in the beta band was directly connected to the higher 


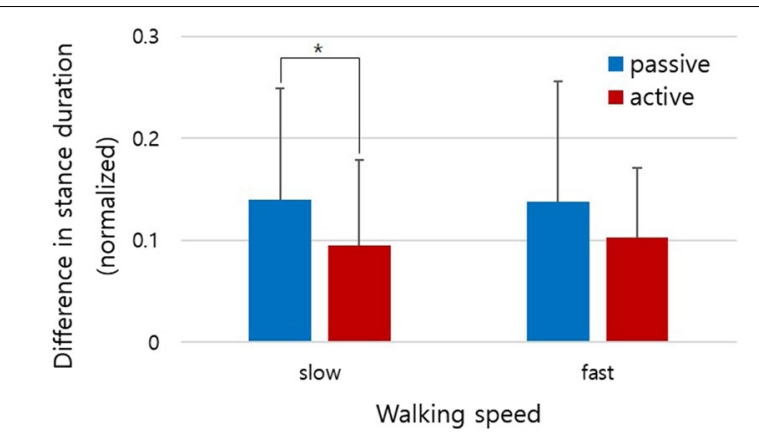

Fig. 6 Gait asymmetries (differences in stance duration) between the passive and active walking tasks from all subjects. All values were normalized to each respective mean stance duration. $\left({ }^{*} p<0.05\right)$

movement selection demand $[59,60]$. Based on such studies, higher activity in the beta band from the sensorimotor cortex during active walking may imply that the suggested active walking system demands a larger population to decide and conduct voluntary movements.

\section{Gait asymmetry decreased}

The increased level of cortical activity levels might be mostly relevant to the swing movement of the affected legs and the simultaneous balance control during the single support phase. Since the participants were asked to match the target walking speed even with the affected lower limb in the stance phase, they needed to lengthen the stance duration with the affected leg, and they were somewhat forced to lengthen the swing movements. In line with this speculation, a recent study reported that self-paced treadmill walking resulted in a larger intrastride walking speed variation and a correspondingly higher risk of falls [61], which probably required additional effort to control balance with the affected side of the lower limb. When the affected leg was in stance during passive walking, subjects showed a $28.8 \%$ decrease in stance duration during the active walking session. In addition, previous studies have revealed that most energy loss and compensation occurred especially during the double support phase [62,63], so sophisticated body control and corresponding high levels of cortical activities were required [28] to maintain body balance and walking speed in the double support phase. If some cortical regions, however, did not have intact control of the affected leg, the healthy leg would share the burden of balancing and propulsion, and gait asymmetry would occur. Higher activation levels and phasic synchronization in the PFC and SG on the affected side of brain during active walking would result in better control when standing, and propulsion would occur. Interestingly, as predicted, the affected leg contacted the ground earlier and propelled the body for a longer duration. As a result, gait asymmetry, defined as a difference in stance duration between the healthy and the affected leg, decreased by approximately $28.8 \%$ from all the subjects during the active walking trials (Fig. 6) compared to the passive walking trials. In addition to the higher cortical activities and phasic correlation found in this study, connectivity between such cortices has to be identified and assessed to ensure that such phasic synchronization and high activation result in improved networking between the cortices and the subsequently reduced gait asymmetry.

Notably, subjects with lesions on the right side of their brain showed significant increases in cortical activation levels but relatively less than the group with the lesion on the opposite side of the brain. First, a source of this inconsistency might have come from the limited number of subjects. In addition, compared to stroke survivors with lesions in their left brain $(13.5 \pm 5.26$ days of stroke onset), the right-side lesion group had a significantly longer onset period (9.18 \pm 13.76 years of stroke onset). It is worth noting that one of the participants (subject \#4 from Table 2) has a significantly longer onset time of stroke of 26 years than other participants, so it may limit a possibility to determine possible effects of timing of gait training after stroke on cortical activities. Earlier timing of the gait rehabilitation might impact cortical activities [64]; however, our study was not able to determine the effects of the rehabilitation timing on the corresponding neuroplasticity due to the limited number of subjects and inconsistent onset time of stroke. and this possibility needs to be further investigated.

\section{CPG}

Although the cortices and their connectivity need to be highly activated to promote neuroplasticity in patients with brain lesions, some previous studies have pointed out that walking at a constant speed, such as passive walking, might impede the development of such connectivity. When a human performs steady walking at a constant speed, a spinal central pattern generator (CPG) would be responsible for the rhythmic movements [6567], so the connectivity between sensorimotor clusters would be diminished [36]. To avoid these low levels of cortical connectivity, transient gait training might be helpful, which could introduce deviations from steadystate for beneficial outcomes. Introducing the target speed to make walkers adjust their gait speeds and to force continuous body control on the user to minimize the error between the target and the real speeds could play a vital role in promoting connectivity in the cortical networks. 


\section{Transient gaits}

In addition to active walking at a target speed, which results in continuous body control, other walking tasks, such as accelerating and decelerating walking and stopping and starting walking, could also be implemented using the self-paced walking training system. In line with the goal of promoting cortical activity and connectivity, for example, such transient gaits could be performed by following a pet that is changing its speed or direction in a virtual reality (VR) system [68]. A variety of gait transients could be performed in a relatively small space and a safe environment at an affordable price because the selfpaced training system consists of only the conventional linear treadmill, a display, and the Kinect. The described self-paced training system, therefore, could be utilized for gait rehabilitation with subjects who have difficulties in being trained outside or low accessibility to rehabilitation systems. In addition, other types of feedback systems can be implemented on the suggested self-paced treadmill walking system as required for gait training after stroke. Rather than the walking speed used in this study, gait asymmetry, for example, can also be provided as feedback to train these features of walking.

\section{Conclusion}

This study focused on the changes in the levels of cortical activity from self-paced treadmill walking. The results should be confined to suggesting the potential of promoting neuroplasticity. Interestingly, not only the intact side but also the affected side showed a significantly higher level of cortical activation, mostly in the $\beta$ and $\gamma$ bands. In addition, to match the given target walking speed with the affected lower limb, the gait asymmetry was also decreased on the self-paced treadmill walking. Thus, it may be possible to promote regaining walking performance even when the affected lower limb is responsible for body control with the self-paced gait training system. To determine if neuroplasticity is promoted using the self-paced walking training system, a long-term clinical test needs to be conducted.

\footnotetext{
Abbreviations

BWSTT: Body weight-supported treadmill training; EEG: Electroencephalography; PMC: Premotor cortex; PFC: Prefrontal cortex; SMC: Supplementary motor cortex; SG: Supramarginal gyrus; SSC: Somatosensory cortex; AG: Angular gyrus; FAC: Functional ambulation categories; BBS: Berg balance scale; FES: Falls Efficacy Scale; 10 mWT: 10-M walking test; GRF: Ground reaction force; HS: Heel-strike; TO: Toe-off; ASR: Artifact subspace reconstruction; EICA: Extended independent component analysis; IC: Independent components; AMICA: Adaptive mixture independent component analysis; ERSP: Event-related spectral perturbations; ERD: Event-related desynchronization; ERS: Event-related synchronization; ACA: Anterior cerebral artery; MCA: Middle cerebral artery; PCA: Posterior cerebral artery.
}

Acknowledgements

Not applicable.

\section{Authors' contributions}

All authors were heavily involved in study design, developing experimental apparatus, conducting an experiment, analyzing data, and writing the manuscript. NP and HP designed this study. HP, KO, SJ, and SH developed the experimental apparatus. KO, SH, SJ, and JP analyzed and interpreted the data. NP and WK performed the clinical examinations. Each of the authors has read and concurs with the content in the final manuscript. The material has not been and will not be submitted for publication elsewhere. All authors read and approved the final manuscript.

\section{Funding}

This work was supported by the National Research Foundation of Korea (NRF) Grant funded by the Korea government (MSIT) (No. NRF-2020R1A2C2012641). This study has also been supported by Grant numbers (16-2016-003, 16-2017004) from the SNUBH-KAIST Collaboration Research Fund.

\section{Availability of data and materials}

Not applicable. But the datasets may be available from the corresponding authors on reasonable request.

\section{Declarations}

\section{Ethics approval and consent to participate}

The institutional review board of SNUBH approved the study protocol (IRB no. B-1608/358-004), and written informed consent was obtained from all the participants.

\section{Consent for publication}

Consents for publication from all the participants were obtained (IRB no. B-1608/358-004).

\section{Competing interests}

The authors have no financial conflicts of interest related to this work.

\section{Author details}

${ }^{1}$ Arms \& Hands Lab, Shirley Ryan AbilityLab, Chicago, IL, USA. ${ }^{2}$ Department of Mechanical Engineering, Korea Advanced Institute of Science and Technology (KAIST), 291 Daehak-ro, Yuseong-gu, Daejeon 34141, Republic of Korea. ${ }^{3}$ Department of Rehabilitation, Seoul National University Bundang Hospital, 82, Gumi-ro 173 Beon-gil, Bundang-gu, Seongnam-si, Gyeonggi-do 13620, Republic of Korea.

Received: 23 November 2020 Accepted: 24 March 2021

Published online: 13 April 2021

\section{References}

1. Liepert J, Bauder H, Miltner WH, Taub E, Weiller C. Treatment-induced cortical reorganization after stroke in humans. Stroke. 2000;31:1210-6.

2. Skilbeck CE, Wade DT, Hewer RL, Wood VA. Recovery after stroke. J Neurol Neurosurg Psychiatry. 1983;46:5-8.

3. Jørgensen HS, Nakayama H, Raaschou HO, Olsen TS. Recovery of walking function in stroke patients: the Copenhagen Stroke Study. Arch Phys Med Rehabil. 1995;76:27-32.

4. Batchelor FA, Mackintosh SF, Said CM, Hill KD. Falls after stroke. Int J Stroke. 2012;7:482-90.

5. Beyaert C, Vasa R, Frykberg GE. Gait post-stroke: pathophysiology and rehabilitation strategies. Clin Neurophysiol. 2015;45:335-55.

6. Törnbom K, Danielsson A. Experiences of treadmill walking with nonimmersive virtual reality after stroke or acquired brain injury - a qualitative study. PLoS ONE. 2018;13:e0209214.

7. Duncan PW, Sullivan KJ, Behrman AL, Azen SP, Wu SS, Nadeau SE, Dobkin $\mathrm{BH}$, Rose DK, Tilson JK, Cen S. Body-weight-supported treadmill rehabilitation after stroke. N Engl J Med. 2011;364:2026-36.

8. Bulea TC, Kim J, Damiano DL, Stanley CJ, Park H-S. User-driven control increases cortical activity during treadmill walking: an EEG study. In: Engineering in medicine and biology society (EMBC), 2014 36th annual international conference of the IEEE. IEEE; 2014. pp. 2111-4. 
9. Yoon J, Park H-S, Damiano DL. A novel walking speed estimation scheme and its application to treadmill control for gait rehabilitation. J Neuroeng Rehabil. 2012;9:62.

10. Chua K, Lim WS, Lim PH, Lim CJ, Hoo CM, Chua KC, Chee J, Ong WS, Liu W, Wong CJ. An exploratory clinical study on an automated, speed-sensing treadmill prototype with partial body weight support for hemiparetic gait rehabilitation in subacute and chronic stroke patients. Front Neurol. 2020;11:747.

11. Kim J, Park H-S, Damiano DL. An interactive treadmill under a novel control scheme for simulating overground walking by reducing anomalous force. IEEE/ASME Trans Mechatron. 2015;20:1491-6.

12. Richards CL, Malouin F, Lamontagne A, McFadyen BJ, Dumas F, Comeau F, Robitaille N-M, Fung J. Gait training after stroke on a self-paced treadmill with and without virtual environment scenarios: a proof-of-principle study. Physiother Can. 2018;70:221-30.

13. Van Dijsseldonk RB, De Jong LA, Groen BE, Vos-Van Der Hulst M, Geurts AC, Keijsers NL. Gait stability training in a virtual environment improves gait and dynamic balance capacity in incomplete spinal cord injury patients. Front Neurol. 2018;9:963.

14. Ray NT, Knarr BA, Higginson JS. Walking speed changes in response to novel user-driven treadmill control. J Biomech. 2018;78:143-9.

15. Kim J, Gravunder A, Stanley CJ, Park H-S. Low-cost implementation of a self-paced treadmill by using a commercial depth sensor. In: Engineering in medicine and biology society (EMBC), 2013 35th annual international conference of the IEEE. IEEE; 2013. pp. 874-7.

16. Canete $\mathrm{S}$, Jacobs DA. Novel velocity estimation for symmetric and asymmetric self-paced treadmill training. J Neuroeng Rehabil. 2021;18:1-15.

17. Choi J-S, Kang D-W, Seo J-W, Tack G-R. Fractal fluctuations in spatiotemporal variables when walking on a self-paced treadmill. J Biomech. 2017;65:154-60.

18. Sloot L, Van der Krogt M, Harlaar J. Self-paced versus fixed speed treadmill walking. Gait Posture. 2014;39:478-84.

19. Wei W, Kaiming Y, Yu Z, Yuyang Q, Chenhui W. A comparison of variability and gait dynamics in spatiotemporal variables between different selfpaced treadmill control modes. J Biomech. 2020;110:109979.

20. Kaur R, Chen Z, Motl R, Hernandez ME, Sowers R. Predicting multiple sclerosis from gait dynamics using an instrumented treadmill — a machine learning approach. IEEE Trans Biomed Eng. 2020. https://doi.org/10.1109/ TBME.2020.3048142.

21. Takeuchi N, Izumi S-I. Rehabilitation with poststroke motor recovery: a review with a focus on neural plasticity. Stroke Res Treat. 2013. https://doi. org/10.1155/2013/128641.

22. Ward NS. Functional reorganization of the cerebral motor system after stroke. Curr Opin Neurol. 2004;17:725-30.

23. Nelles $G$, Jentzen $W$, Jueptner M, Müller S, Diener H. Arm training induced brain plasticity in stroke studied with serial positron emission tomography. Neuroimage. 2001;13:1146-54.

24. Liepert J, Uhde I, Gräf S, Leidner O, Weiller C. Motor cortex plasticity during forced-use therapy in stroke patients: a preliminary study. J Neurol. 2001;248:315-21.

25. La Fougere C, Zwergal A, Rominger A, Förster S, Fesl G, Dieterich M, Brandt T, Strupp M, Bartenstein P, Jahn K. Real versus imagined locomotion: a [18F]-FDG PET-fMRI comparison. Neuroimage. 2010;50:1589-98.

26. Miyai I, Tanabe HC, Sase I, Eda H, Oda I, Konishi I, Tsunazawa Y, Suzuki T, Yanagida T, Kubota K. Cortical mapping of gait in humans: a near-infrared spectroscopic topography study. Neuroimage. 2001;14:1186-92.

27. Suzuki M, Miyai I, Ono T, Oda I, Konishi I, Kochiyama T, Kubota K. Prefrontal and premotor cortices are involved in adapting walking and running speed on the treadmill: an optical imaging study. Neuroimage. 2004;23:1020-6.

28. Gwin JT, Gramann K, Makeig S, Ferris DP. Electrocortical activity is coupled to gait cycle phase during treadmill walking. Neuroimage. 2011;54:1289-96.

29. Koenraadt KL, Roelofsen EG, Duysens J, Keijsers NL. Cortical control of normal gait and precision stepping: an fNIRS study. Neuroimage. 2014:85:415-22.

30. Doi T, Makizako H, Shimada H, Park H, Tsutsumimoto K, Uemura K, Suzuki T. Brain activation during dual-task walking and executive function among older adults with mild cognitive impairment: a fNIRS study. Aging Clin Exp Res. 2013;25:539-44.
31. Pfurtscheller G, Da Silva FL. Event-related EEG/MEG synchronization and desynchronization: basic principles. Clin Neurophysiol. 1999;1 10:1842-57.

32. Seeber M, Scherer R, Wagner J, Solis-Escalante T, Müller-Putz GR. EEG beta suppression and low gamma modulation are different elements of human upright walking. Front Hum Neurosci. 2014;8:485.

33. Bulea TC, Kim J, Damiano DL, Stanley CJ, Park H-S. Prefrontal, posterior parietal and sensorimotor network activity underlying speed control during walking. Front Hum Neurosci. 2015;9:247.

34. Seth AK, Barrett AB, Barnett L. Granger causality analysis in neuroscience and neuroimaging. J Neurosci. 2015;35:3293-7.

35. Friston K, Moran R, Seth AK. Analysing connectivity with Granger causality and dynamic causal modelling. Curr Opin Neurobiol. 2013;23:172-8.

36. Lau TM, Gwin JT, Ferris DP. Walking reduces sensorimotor network connectivity compared to standing. J Neuroeng Rehabil. 2014;11:14.

37. Holden MK, Gill KM, Magliozzi MR, Nathan J, Piehl-Baker L. Clinical gait assessment in the neurologically impaired: reliability and meaningfulness. Phys Ther. 1984;64:35-40.

38. Holden MK, Gill KM, Magliozzi MR. Gait assessment for neurologically impaired patients: standards for outcome assessment. Phys Ther. 1986:66:1530-9.

39. Berg K, Wood-Dauphine S, Williams J, Gayton D. Measuring balance in the elderly: preliminary development of an instrument. Physiother Can. 1989:41:304-11.

40. An S, Sin H, Cho H, Lee G. The reliability and validity of the falls efficacy scale (Korean version) in stroke patients. J Spec Educ Rehabil Sci. 2012;51:363-81.

41. Mullen T, Kothe C, Chi YM, Ojeda A, Kerth T, Makeig S, Cauwenberghs G, Jung T-P. Real-time modeling and $3 \mathrm{D}$ visualization of source dynamics and connectivity using wearable EEG. In: Engineering in medicine and biology society (EMBC), 2013 35th annual international conference of the IEEE. IEEE; 2013. pp. 2184-7.

42. Jung T-P, Humphries C, Lee T-W, Makeig S, McKeown MJ, Iragui V, Sejnowski TJ. Extended ICA removes artifacts from electroencephalographic recordings. In: Advances in neural information processing systems. 1998. pp. 894-900.

43. Delorme A, Makeig S. EEGLAB: an open source toolbox for analysis of single-trial EEG dynamics including independent component analysis. J Neurosci Methods. 2004;134:9-21.

44. Zhou W, Gotman J. Removal of EMG and ECG artifacts from EEG based on wavelet transform and ICA. In: Engineering in medicine and biology society, 2004 IEMBS'04 26th annual international conference of the IEEE. IEEE; 2004. pp. 392-5.

45. Oostenveld R, Oostendorp TF. Validating the boundary element method for forward and inverse EEG computations in the presence of a hole in the skull. Hum Brain Mapp. 2002;17:179-92.

46. Lancaster JL, Woldorff MG, Parsons LM, Liotti M, Freitas CS, Rainey L, Kochunov PV, Nickerson D, Mikiten SA, Fox PT. Automated Talairach atlas labels for functional brain mapping. Hum Brain Mapp. 2000;10:120-31.

47. Mihara M, Miyai I, Hatakenaka M, Kubota K, Sakoda S. Role of the prefrontal cortex in human balance control. Neuroimage. 2008;43:329-36.

48. Miller EK, Cohen JD. An integrative theory of prefrontal cortex function. Annu Rev Neurosci. 2001;24:167-202.

49. Goldberg G. Supplementary motor area structure and function: review and hypotheses. Behav Brain Sci. 1985;8:567-88.

50. Dick J, Benecke R, Rothwell J, Day B, Marsden C. Simple and complex movements in a patient with infarction of the right supplementary motor area. Mov Disord. 1986;1:255-66.

51. Lee K-M, Chang K-H, Roh J-K. Subregions within the supplementary motor area activated at different stages of movement preparation and execution. Neuroimage. 1999;9:117-23.

52. Jacobs JV, Lou J-S, Kraakevik JA, Horak FB. The supplementary motor area contributes to the timing of the anticipatory postural adjustment during step initiation in participants with and without Parkinson's disease. Neuroscience. 2009;164:877-85.

53. Kheradmand A, Lasker A, Zee DS. Transcranial magnetic stimulation (TMS) of the supramarginal gyrus: a window to perception of upright. Cereb Cortex. 2015;25:765-71.

54. Andres M, Pelgrims B, Olivier E, Vannuscorps G. The left supramarginal gyrus contributes to finger positioning for object use: a neuronavigated transcranial magnetic stimulation study. Eur J Neurosci. 2017;46:2835-43. 
55. Richer F, Martinez M, Robert M, Bouvier G, Saint-Hilaire J-M. Stimulation of human somatosensory cortex: tactile and body displacement perceptions in medial regions. Exp Brain Res. 1993;93:173-6.

56. Kuo C-C, Luu P, Morgan KK, Dow M, Davey C, Song J, Malony AD, Tucker DM. Localizing movement-related primary sensorimotor cortices with multi-band EEG frequency changes and functional MRI. PLOS ONE. 2014;9:e112103.

57. Pfurtscheller G. Central beta rhythm during sensorimotor activities in man. Electroencephalogr Clin Neurophysiol. 1981;51:253-64.

58. Chung JW, Ofori E, Misra G, Hess CW, Vaillancourt DE. Beta-band activity and connectivity in sensorimotor and parietal cortex are important for accurate motor performance. Neuroimage. 2017;144:164-73.

59. Brinkman L, Stolk A, Marshall TR, Esterer S, Sharp P, Dijkerman HC, de Lange FP, Toni I. Independent causal contributions of alpha-and beta-band oscillations during movement selection. J Neurosci. 2016;36:8726-33.

60. Brinkman L, Stolk A, Dijkerman HC, de Lange FP, Toni I. Distinct roles for alpha-and beta-band oscillations during mental simulation of goaldirected actions. J Neurosci. 2014;34:14783-92.

61. Qian Y, Yang K, Zhu Y, Wang W, Wan C. Local dynamic stability of selfpaced treadmill walking versus fixed-speed treadmill walking. J Biomech Eng. 2020. https://doi.org/10.1115/1.4045595.
62. Donelan JM, Kram R, Kuo AD. Mechanical work for step-to-step transitions is a major determinant of the metabolic cost of human walking. J Exp Biol. 2002;205:3717-27.

63. Donelan JM, Kram R, Kuo AD. Simultaneous positive and negative exter nal mechanical work in human walking. J Biomech. 2002;35:117-24.

64. Maulden SA, Gassaway J, Horn SD, Smout RJ, DeJong G. Timing of initiation of rehabilitation after stroke. Arch Phys Med Rehabil. 2005:86:34-40.

65. Zehr EP, Duysens J. Regulation of arm and leg movement during human ocomotion. Neuroscientist. 2004;10:347-61.

66. Marder E, Calabrese RL. Principles of rhythmic motor pattern generation. Physiol Rev. 1996;76:687-717.

67. Dimitrijevic MR, Gerasimenko Y, Pinter MM. Evidence for a spinal central pattern generator in humans. Ann N Y Acad Sci. 1998;860:360-76.

68. Oh K, Stanley CJ, Damiano DL, Kim J, Yoon J, Park H-S. Biomechanical evaluation of virtual reality-based turning on a self-paced linear treadmill. Gait Posture. 2018:65:157-62.

\section{Publisher's Note}

Springer Nature remains neutral with regard to jurisdictional claims in published maps and institutional affiliations.
Ready to submit your research? Choose BMC and benefit from:

- fast, convenient online submission

- thorough peer review by experienced researchers in your field

- rapid publication on acceptance

- support for research data, including large and complex data types

- gold Open Access which fosters wider collaboration and increased citations

- maximum visibility for your research: over $100 \mathrm{M}$ website views per year

At BMC, research is always in progress.

Learn more biomedcentral.com/submissions 